\title{
Stimulation of chondrocytes and chondroinduced mesenchymal stem cells by osteoinduced mesenchymal stem cells under a fluid flow stimulus on an integrated microfluidic device
}

\author{
XUANWEN BAO ${ }^{1,2}$, ZHONGYU LI ${ }^{1}$, HUI LIU ${ }^{1}$, KE FENG $^{1}$, FANGCHAO YIN ${ }^{1}$, HONGJING LI ${ }^{2}$ and JIANHUA QIN ${ }^{1}$ \\ ${ }^{1}$ Department of Biotechnology, Dalian Institute of Chemical Physics, Chinese Academy of Sciences, Dalian, Liaoning 116023; \\ ${ }^{2}$ Department of Orthopaedics, The First Affiliated Hospital of Dalian Medical University, Dalian, Liaoning 116000, P.R. China
}

Received September 8, 2017; Accepted November 9, 2017

DOI: $10.3892 / \mathrm{mmr} .2017 .8153$

\begin{abstract}
The aim of the present study was to investigate the stimulation of osteoinduced mesenchymal stem cells (MSCs) into chondrogenically predifferentiated MSCs and chondrocytes in a mechanical environment. A novel two-layer microfluidic chip was used to mimic the interstitial flow in the superficial zones of articular cartilage. The morphology, proliferation rate and the expression of collagen I, collagen II and aggrecan of chondrocytes and chondro-MSCs were investigated. The results revealed that the cells in the bottom layer were influenced by the top layer's osteoinduced MSCs and the bottom layer's shear flow. The expression of collagen I, which may signify the effect of the shear stress on the dedifferentiation change, was weakened by the stimulation of osteoinduced MSCs on the top layer. The expression of collagen II and aggrecan was increased in the fluidic environment by osteoinduced MSCs. These results indicate that osteoinduced MSCs have a significant effect on the phenotype of chondro-MSCs and chondrocytes in the fluidic microenvironment. The present study described a simple and promising way to rapidly evaluate cell responses to other cells in a fluidic environment, which may help to better promote the utilization of MSCs and chondrocytes in tissue engineering.
\end{abstract}

\section{Introduction}

Osteoarthritis has been one of the most popular diseases that afflict many people in the aging population. Currently, there

Correspondence to: Dr Hongjing Li, Department of Orthopaedics, The First Affiliated Hospital of Dalian Medical University, 222 Zhongshan Road, Dalian, Liaoning 116000, P.R. China

E-mail:1hj68430@163.com

Dr Jianhua Qin, Department of Biotechnology, Dalian Institute of Chemical Physics, Chinese Academy of Sciences, 457 Zhongshan Road, Dalian, Liaoning 116023, P.R. China

E-mail: jhqin@dicp.ac.cn

Key words: microfluidics, mesenchymal stem cells, shear stress, intercellular interactions, metabolism is no effective therapy for the treatment of osteoarthritis due to the inferior intrinsic repair capacity of the damaged area of cartilage. Surgeons often use palliative measures to relieve the symptoms. However, the pathophysiological change during the disease progression cannot be restored by palliative treatments. Thus, the final solution is the joint replacement surgery. The development of tissue engineering provides new insights into the treatment of osteoarthritis. 3D culture of mesenchymal stem cells (MSCs) and chondrocytes in tissue engineering can reduce cell dedifferentiation $(1,2)$, which have promoted the autologous implantation technology into the clinical application $(3,4)$. Since the interactions between bone and cartilage sides are central to the progression of osteoarthritis, many researchers consider osteoarthritis as a disease of osteochondral tissue. Thus, several bi-phasic scaffolds were investigated to anchor the cartilage phase of scaffold to integrate better into the lesion area (5-7).

Since MSCs have great potential to proliferate and differentiate into osteogenic and chondrogenic phenotypes in a desired physiological environment, they are promising cell resource for osteochondral tissue engineering (8-10). In the bone and cartilage phases of scaffold, MSCs differentiate into osteogenic lineage and chondrogenic lineage, respectively. The different lineages of cells in the bi-phasic scaffold might have an interaction through cytokine secretion. In the previous studies, MSCs have showed the ability to secrete a wide range of bioactive factors, e.g., matrix metalloproteinase, tissue inhibitor of metalloproteinases, transforming growth factor (TGF)- $\beta 1$ and fibroblast growth factor-2 (11-14), to influence cell behavior and tissue remodeling. However, there is still controversy about the interactions between cells in bi-phasic scaffold. Some researchers have studied the interactions between osteoinduced MSCs and chondroinduced MSCs or interactions between osteoinduced MSCs and chondrocytes under static conditions, which cann't mimic the fluidic environment in vivo (15-17). To enhance the therapeutic potential of MSCs and chondrocytes in the treatment of osteoarthritis and other degenerative joint diseases, it is meaningful to investigate the stimulation of cells in the upper layer of bi-phasic scaffold to cells on the bottom layer in a fluidic microenvironment which can better mimic the mechanical characteristics in vivo. 
The specific goals of this study were to investigate the effects of osteoinduced MSCs, fluidic shear stress, or the combination of them on the phenotype of chondroinduced MSCs and chondrocytes, which can help illustrate the interaction of cells on double layer in fluidic environment. Herein, we designed a novel integrated lab-on-a-chip to introduce cells and perfusion systems and thus mimic the fluidic flow in vivo. By using perfusion systems and a semipermeable membrane, we studied the individual and the combination effect of osteoinduced MSCs and shear stress on the phenotype of chondroinduced MSCs and chondrocytes. Through the computational fluid dynamics (CFD) method, we simulated the 3D flow field and obtained a stable shear stress in the cell chambers. The semipermeable membrane allows the transition of cytokines and thus helps us to study the interactions between osteoinduced MSCs and other related cells (chondrocytes, chondroinduced MSCs). The three chambers on the bottom layer allow us to study the influence of cells on the up layer to cells in three chambers separately at one time. In a word, we designed a novel microfludic chip and decreased the size of experimental device to micrometer level and reduced cell numbers needed in one experiment, which may be helpful to future clinical translation research.

\section{Materials and methods}

Isolation and culture of MSCs and chondrocytes. All the experiments were approved by the Committee on Animal Use and Care of the Dalian Medical University. The rats were euthanized prior to the collection of tissue samples. Articular cartilage chondrocytes were isolated from humeral heads, femoral heads, and femoral condyles of male Sprague-Dawley rats weighing 80-120 g, as reported (18). Briefly, chondrocytes were isolated by digestion with $0.15 \%$ type II collagenase for $16 \mathrm{~h}$ and resuspended in the Dulbecco's modified Eagle's medium/F-12 (HyClone; GE Healthcare Life Sciences, Logan, UT, USA) containing 10\% fetal bovine serum (FBS; HyClone; GE Healthcare Life Sciences), $50 \mathrm{mg} / \mathrm{ml}$ ascorbic acid-2-phosphate (Sigma-Aldrich; Merck KGaA, Darmstadt, Germany), and $100 \mathrm{U} / \mathrm{ml}$ penicillin-streptomycin. The primary chondrocytes obtained were used in the subsequent experiments.

The primary rat MSCs were isolated from the bilateral femurs and tibias at the same time. After the distal ends of the bone were cut open, the marrow cavities were lavaged with sterile phosphate-buffered saline (PBS). The cells were then resuspended in the high-glucose Dulbecco's modified Eagle's medium (DMEM; Gibco; Thermo Fisher Scientific, Inc., Waltham, MA, USA) containing 10\% FBS (HyClone; GE Healthcare Life Sciences) and $100 \mathrm{U} / \mathrm{ml}$ penicillin-streptomycin. After incubation at $37^{\circ} \mathrm{C}$ in $5 \% \mathrm{CO}_{2}$ for $48 \mathrm{~h}$, the medium was changed to remove the nonadherent cells. After two passages, the attached MSCs were then used in the following experiments.

The microdevice was coated with $100 \mathrm{mg} / \mathrm{ml}$ fibronectin (Sigma-Aldrich; Merck KGaA) for $1 \mathrm{~h}$ at room temperature. Then, the chambers were washed with PBS. The suspension of osteoinduced MSCs at a density of $1 \times 10^{5}$ cells $/ \mathrm{ml}$ was injected gently from the inlet of top chamber to seed cells on the polycarbonate membrane. After $12 \mathrm{~h}$, a chondroinduced
MSCs suspension of $0.5 \times 10^{5}$ cells $/ \mathrm{ml}$, a chondrocyte suspension of $1 \times 10^{5}$ cells $/ \mathrm{ml}$ and a mixture of two cells with the corresponding cell density were injected into the three microchambers, respectively. The chip was incubated at $37^{\circ} \mathrm{C}$ for $12 \mathrm{~h}$ to allow the cell attachment. In groups 2 and 4 , the inlets of the bottom layer were connected to three peristaltic pumps (Longer Pump BT100-2J; Longer Precision Pump Co., Ltd., Baoding, China). The cells were exposed to $1 \mathrm{mmol} / 1$ cytochalasin D (CytoD; Sigma-Aldrich; Merck KGaA) by pipetting medium containing CytoD into the inlet of chip for $1 \mathrm{~h}$ to disrupt stress fibers before the application of the flow stimulus.

In vitro induction of lineage differentiation. To generate chondrogenic cells, MSCs were first expanded for 5 days and then subjected to 14 days of predifferentiation in a serum-free chondrogenic medium containing DMEM-LG, $6.25 \mu \mathrm{g} / \mathrm{ml}$ insulin, $50 \mathrm{mg} / 1$ ascorbic acid, $1 \times 10^{-7} \mathrm{~mol} / 1$ dexamethasone, 10 ng/ml TGF- 33 (PeproTech, Inc., Rocky Hill, NJ, USA) and $1 \mu \mathrm{M}$ ascorbate-2-phosphate (Wako Chemicals USA, Inc., Richmond, VA, USA), 1\% sodium pyruvate (Invitrogen; Thermo Fisher Scientific, Inc.). To obtain Osteogenic commitment MSCs, MSCs were cultured in a complete osteogenic medium containing high glucose DMEM, $10 \%$ (v/v) FBS, $50 \mathrm{mg} / \mathrm{l}$ ascorbic acid, $10 \mathrm{mM} \beta$-glycerophosphate, $1 \times 10^{-8} \mathrm{~mol} / 1$ dexamethasone. Osteoinduced MSCs were exposed to the osteogenic medium for 7 days.

Fabrication of microfluidic chip. The schematic diagram of the device is as shown in Fig. 1A. The device was composed of two layers of polydimethylsiloxane (PDMS) with several microchambers. The top layer of the chip has one cell culture chamber which was $500 \mu \mathrm{m}$ in height, $6 \mathrm{~mm}$ in width and $11 \mathrm{~mm}$ in length and the bottom layer has three microchambers, each of which was $500 \mu \mathrm{m}$ in height, $2 \mathrm{~mm}$ in width and $16 \mathrm{~mm}$ in length. Between two pieces of PDMS, there was a polycarbonate membrane (Whatman Inc., Piscataway, NJ, USA), with the pore size of $0.4 \mu \mathrm{m}$, which allowed the transition of cytokines and small molecular compounds (19). Each chamber has one inlet and one outlet, respectively. Perfusion system could be linked with the inlet of each chamber to provide the fluid flow.

Microfluidic chip was fabricated using a conventional microfabrication technique. The mask was designed by AutoCAD 2007 (Autodesk, Inc., San Rafael, CA, USA) and printed on transparencies with 4,000 dpi resolution. The master was fabricated by photoresist SU-8 3035 through soft lithography technique. Then the microfluidic chip was fabricated in PDMS by replicating molding the master, followed by the curing process of PDMS at $80^{\circ} \mathrm{C}$ in an oven for one hour. After that, PDMS layer was peeled off from the master. A polycarbonate membrane and two pieces of PDMS were sealed together after the treatment by oxygen plasma for $1 \mathrm{~min}$.

Experimental design. The overall experimental design is as shown in Fig. 2. In this study, the top layer of the chip was seeded with or without osteoinduced MSCs, and the bottom layer of the chip was separately seeded with chondroinduced MSCs, chondrocytes and a mixture of them in three microchambers. MSCs were first expanded for 14 days 
A
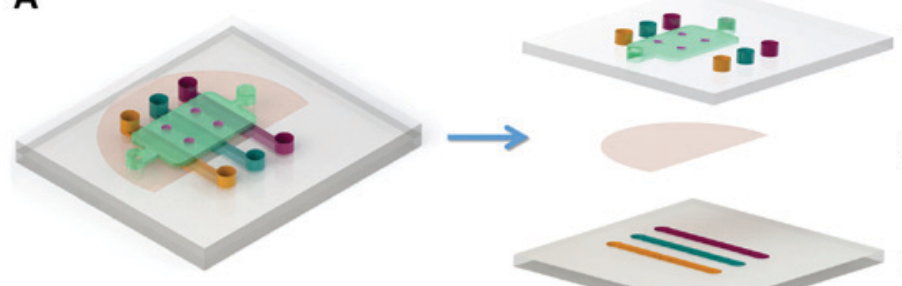

Top layer

The Chip

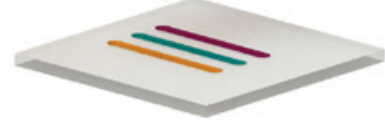

\section{Semipermeable membrane}

Bottom layer

B
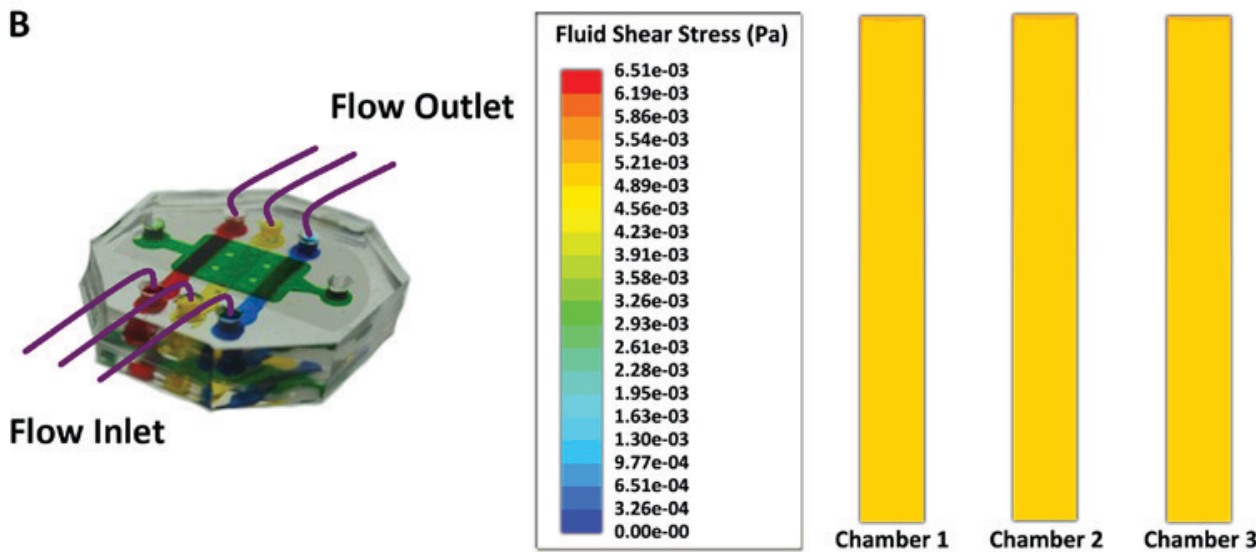

Figure 1. (A) The schematic diagram of the two-layer microfluidic device. The microfluidic chip is composed of top and bottom layers. There is a semipermeable membrane between the two layers. (B) The numerical simulation results for the cell culture chambers based on computational fluid dynamics analysis. Contours of the wall shear stress distribution in the microchambers of the device. The central area of the chambers exhibited a uniform shear stress distribution within the wall, and the average shear stress of the bottom wall was approximately 0.05 dyne $/ \mathrm{cm}^{2}$, which is equal to the interstitial fluid flow in the cartilage space $\left(1\right.$ dyne $/ \mathrm{cm}^{2}=0.1$ pascals).
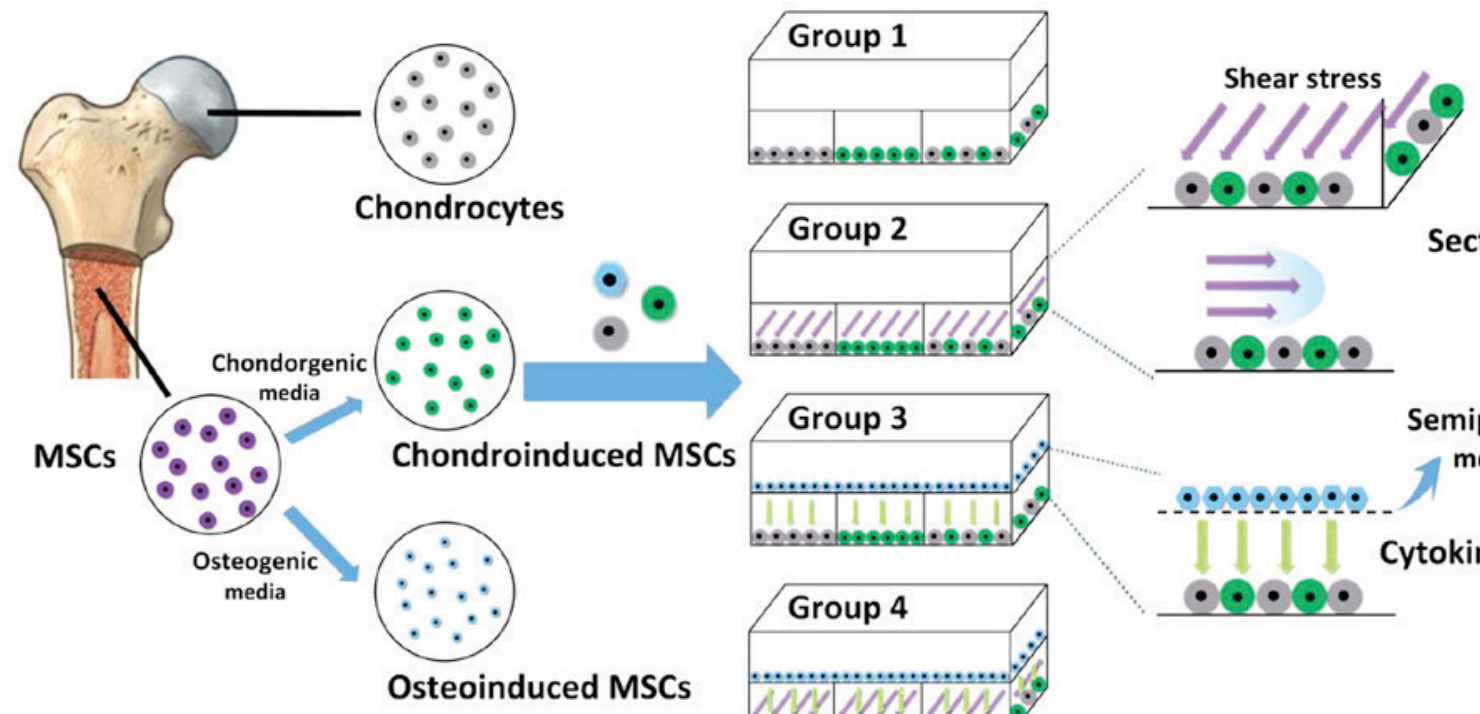

Section view

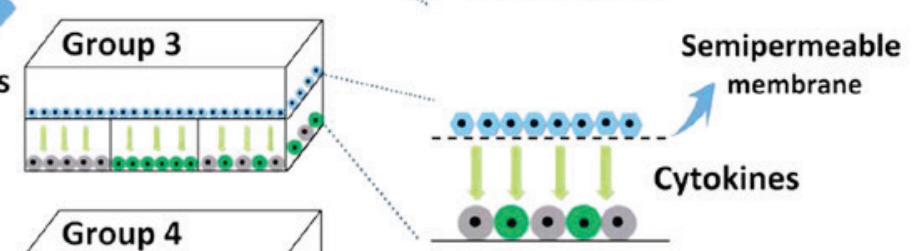

Figure 2. Schematic representation of the overall experimental design. According to the outlined experimental flowchart, MSCs were cultured in the chondrogenic medium for 14 days after 5 days of general expansion. MSCs were cultured in the complete osteogenic medium for 7 days. Cells were then seeded accordingly. After that, the microfluidic chips with cells loading were cultured for 7 days. MSCs, mesenchymal stem cells.

in the general medium and then incubated in the osteogenic medium for another 7 days to obtain osteogenic phenotype cells. Chondroinduced MSCs were cultured for 14 days in the chondrogenic medium with TGF- $\beta 3$ to obtain chondroinduced MSCs. After 14 days' culture, the chondroinduced MSCs were seeded on the bottom of the chip alone or co-cultured with chondrocytes.
Numerical modeling of the shear stress. To produce a certain shear stress $\left(0.05\right.$ dyne $\left./ \mathrm{cm}^{2}\right)$ in the microchambers on the bottom layer of the chip, three perfusion pumps were connected with the inlets of the three microchambers. The cells cultured there were subjected to the shear stress simultaneously. We assumed the flow as a model of laminar and incompressible fluid. A finite volume method (FVM)-based CFD could 
simulate the 3D flow field in the microchambers with a known pressure at the inlet and outlet of the microchamber. To avoid the computational rigor required to solve Fourier series expansions, we used an approximate version in algebraic form as follows: $\mathrm{R}=[12 \eta \mathrm{L} /(1-0.63(\mathrm{~h} / \mathrm{w})] \times(1 / \mathrm{h} 3 \mathrm{w})$.

In this formula, $R$ is the hydraulic resistance of the rectangular microchambers, $\eta$ the dynamic viscosity of the liquid, $L$ the channel length, $h$ and $w$ (always $h<w$ ) the channel height and width, respectively. With a given perfusion rate, the $\Delta p$ could be obtained and used in CFD method. This result can be summarized using the Hagen-Poiseuille equation, as follows: $\Delta \mathrm{p}=\mathrm{QR}_{H}$.

After some calculations, the pressure data obtained were then used as the inlet and outlet pressure conditions to simulate the 3D flow field in each culture chamber using the CFD method. The computational domain was discretized using approximately 52,000 hexahedral meshes and solved using FVM along with the aforementioned inlet/outlet pressure conditions and no-slip boundary conditions at the chamber walls. The density of the perfusion medium was $993.2 \mathrm{~kg} / \mathrm{m}^{3}$, and the viscosity was $7.85 \times 10^{-4} \mathrm{~Pa} \cdot \mathrm{s}$ at $37^{\circ} \mathrm{C}$.

Colony formation. Cells were seeded at a density of 60 cells $/ \mathrm{cm}^{2}$ and cultured for 14 days. Then the cells were washed with PBS and fixed with acetone/methanol (1:1). 2\% crystal violet solution was used for 20 min staining. Excess stain was removed with tap water.

Alkaline phosphatase staining and Alcian Blue assay. After MSCs were exposed to the osteogenic medium for 7 days, the osteogenic differentiation was detected by alkaline phosphatase (ALP) activity. After a wash with PBS, the cells were fixed with $500 \mu \mathrm{l}$ of $4 \%$ paraformaldehyde for $2 \mathrm{~min}$. Fixed cells were washed with $1 \mathrm{ml}$ of washing buffer $(0.05 \%$ Tween-20 in PBS w/o calcium and magnesium) and incubated with BCIP ${ }^{\circledR} / \mathrm{NBT}$ substrate at RT for $10 \mathrm{~min}$. After a wash cells staining for alkaline phosphatase activity were observed under a microscope.

The chondrogenic differentiation was detected by Alcian Blue Assay. After being fixed for 15 min with $4 \%$ glutaraldehyde at room temperature, the cells were rinsed with $0.1 \mathrm{~N}$ $\mathrm{HCl}$ to decrease the $\mathrm{pH}$ to 1.0 , stained 30 min with $1 \%$ Alcian blue solution (Sigma-Aldrich; Merck KGaA), and rinsed twice with $0.1 \mathrm{~N} \mathrm{HCl}$ to remove nonspecific staining.

Live cell staining. The live cells on the bottom layer were stained by plasma membrane dye DiI (Invitrogen; Thermo Fisher Scientific, Inc.) and DiO (Invitrogen; Thermo Fisher Scientific, Inc.) to illustrate the distribution of cells in the three chambers. The cells were exposed to $10 \mu \mathrm{M}$ DiI and DiO for $1 \mathrm{~h}$ and then cells were rinsed with fresh medium before pipetting into the inlet of chip.

Cell staining and image assay. The vimentin intermediate filament, collagen I, collagen II and aggrecan were stained by immunofluorescence. Chondroinduced MSCs and chondrocytes were fixed in a $4 \%$ paraformaldehyde solution for $15 \mathrm{~min}$, followed by permeabilization with $0.1 \%$ Triton-X for $10 \mathrm{~min}$. After being washed with PBS for 3 times, the samples were blocked with goat serum
Table I. Sequences of primers used in reverse transcription-quantitative polymerase chain reaction.

\begin{tabular}{llr}
\hline Gene & \multicolumn{1}{c}{$\begin{array}{c}\text { PCR primer } \\
\text { sequences (5'-3') }\end{array}$} & $\begin{array}{c}\text { GenBank } \\
\text { accession }\end{array}$ \\
\hline Collagen I & $\begin{array}{l}\text { Forward CCCTGGTCTTG } \\
\text { GAGGAAACT }\end{array}$ & NM_053304 \\
& $\begin{array}{l}\text { Reverse GCACGGAAACT } \\
\text { CCAGCTGAT }\end{array}$ & \\
Collagen II & Forward GGAAACTTTGC & NM_012929 \\
& AGCCCAGAT & \\
& Reverse GCCTTGCATGA & \\
Aggrecan & CTCCCATC & \\
& Forward TGATGCATGGC & NM_022190 \\
& ATTGAGGAC & \\
& Reverse TCGGTCAAAGT & \\
& CCAGTGTG & \\
& Forward CAGCCATGTAC & NM_031144 \\
& GTAGCCATC & \\
& Reverse GTCTCCGGAGT & \\
& CCATCACAA
\end{tabular}

for $30 \mathrm{~min}$ at room temperature and incubated with anti-intermediate filament (BIOSYNTHESIS, China), anti-collagen II (Beijing Biosynthesis Biotechnology Co., Ltd., Beijing, China), anti-collagen I (Beijing Biosynthesis Biotechnology Co., Ltd.) and anti-aggrecan (Beijing Biosynthesis Biotechnology Co., Ltd.) overnight. Then they were mixed with FITC-conjugated goat anti-rabbit IgG or TRITC-conjugated goat anti-rabbit secondary antibodies (Zhongshan Golden Bridge, Beijing, China) for $1 \mathrm{~h}$ at room temperature, respectively. Cell nuclei were stained with 40, 6-diamidino-2-phenylindole (DAPI) for the following $10 \mathrm{~min}$. For the imaging of cell shape, the intermediate filament was stained by using the same protocol. After staining, the chip was washed with PBS for 2-3 times. The cells were then observed on an Olympus fluorescence microscopy (Olympus IX 71; Olympus Corporation, Tokyo, Japan Japan). The images were analyzed using image Pro Plus 6.0 software. The angle of orientation and the fluorescence area per cell were analyzed by the intermediate filament staining with Image $\mathbf{J}$ to evaluate the effect of shear stress.

Living and dead cells were distinguished by different colors. Propidium iodide visualise the nucleus and more than 100 nucleus in each group were picked randomly to check cellular viability. For cell proliferation analysis, cells were fixed and stained by DAPI to count the proliferation rate in each group.

Quantitative PCR ( $q P C R)$. After 7 days exposure to fluid flow stimulus, qPCR was used to analyse the expression level of collagen I, collagen II and aggrecan. After rinsing with cold PBS once, Total RNA was extracted from the cultured cells with TRIzol (Invitrogen; Thermo Fisher Scientific, Inc.), and first-strand complementary cDNA was synthesized using the PrimeScript RT reagent kit (Takara Biotechnology Co., Ltd., Dalian, China). qPCR was performed using Power 

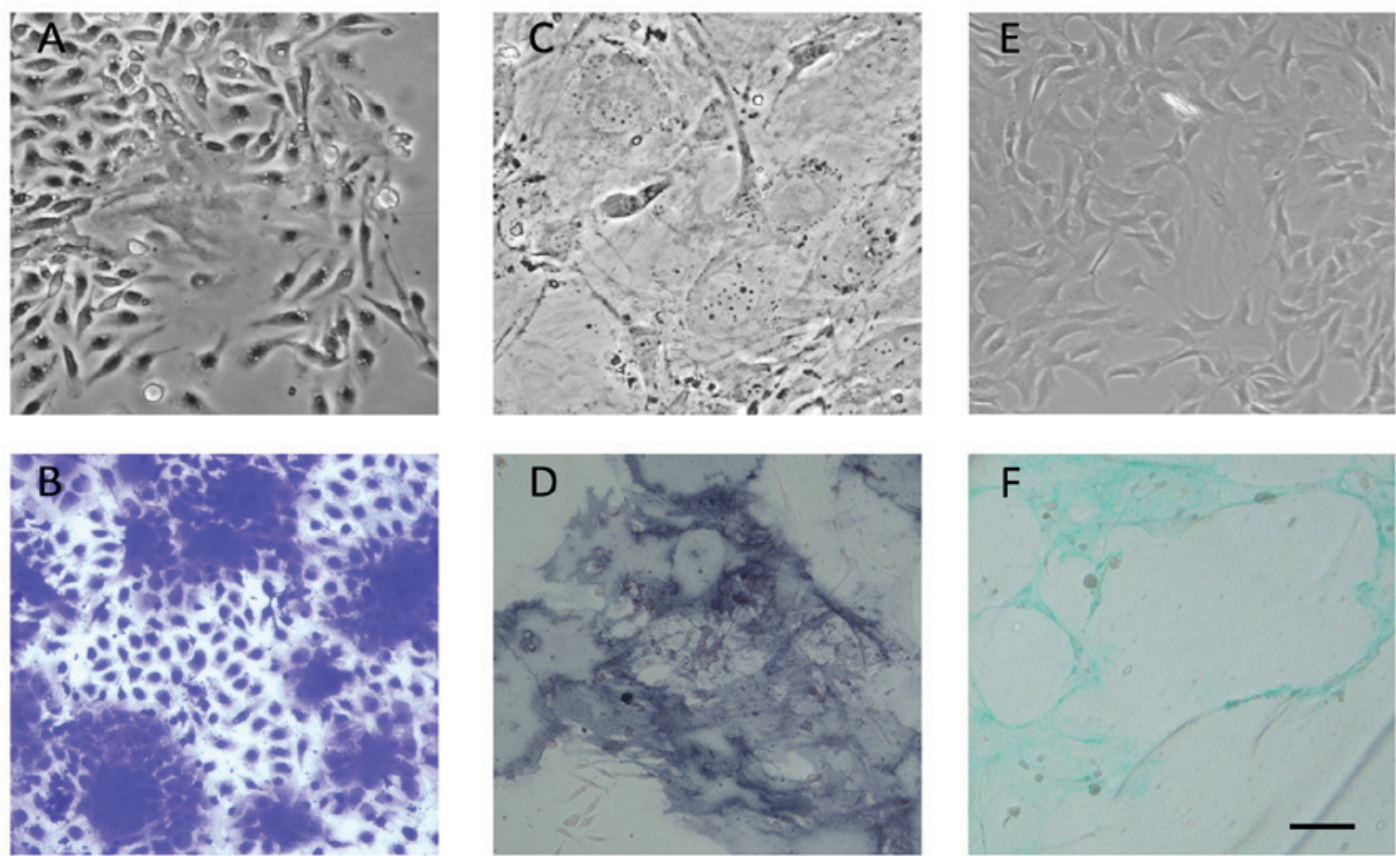

Figure 3. Phenotype of isolated MSCs and induced MSCs. (A) Bright field picture of primary MSCs. (B) Colony formation capacity was showed by Crystal violet staining. (C) Bright field picture of osteogenic commitment MSCs. (D) The osteogenic commitment differentiation was proved by alkaline phosphatase staining. (E) Bright field picture of chondroinduced MSCs. (F) The chondroinduced differentiation was proved by Alcian Blue staining. Scale bar is $100 \mu \mathrm{m}$. MSCs, mesenchymal stem cells.

SYBR-Green PCR Master Mix (Applied Biosystems; Thermo Fisher Scientific, Inc.) in combination with a 7500 Real-Time PCR Detection System. $\beta$-actin was used as the internal control gene to normalize the quantities of target gene expressions. Thermocycling conditions were as follows: $95^{\circ} \mathrm{C}$ for $30 \mathrm{sec} 40$ cycles of denaturation $\left(95^{\circ} \mathrm{C}, 5 \mathrm{sec}\right)$, annealing $\left(60^{\circ} \mathrm{C}\right.$, $30 \mathrm{sec})$ and extension $\left(72^{\circ} \mathrm{C}, 30 \mathrm{sec}\right)$. The primers used in this study were listed in Table I. The relative mRNA level was expressed as fold change relative to group 1 after normalization to the expression of $\beta$-actin with $2^{-\Delta \Delta \mathrm{Cq}}$ method (20).

Statistical analysis. In this study, all the experiments were performed at least in triplicate with different batches of microfluidic chips. All the results were presented as mean \pm standard deviation. One-way ANOVA method followed by Tukey's post hoc test was used to compare the statistically significant differences among different groups. $\mathrm{P}<0.05$ was considered to indicate statistical significance.

\section{Results}

Computational simulation of fluid shear stress. In this study, peristaltic pumps were connected the inlets of the microchambers to drive the fluid flow through the bottom layer of chip. The fluid flow was Poiseuille flow and we assumed that the shear stress on the cells was equal to the shear stress of the bottom wall of the chamber. When a perfusion flow rate of $7 \mu \mathrm{l} / \mathrm{h}$ was applied to the microchip, a constant fluid flow stimulus could be generated on the cells. From the simulation results by FVM-based CFD (Fig. 1B), the central area of the chambers exhibited a uniform shear stress distribution within the wall (the uniform yellow color means a uniform 0.05 dyne $/ \mathrm{cm}^{2}$ shear stress in three chambers, $1 \mathrm{dyne} / \mathrm{cm}^{2}=0.1$ pascals), and the average shear stress of the bottom wall was approximately $0.05 \mathrm{dyne} / \mathrm{cm}^{2}$, which is equal to the interstitial fluid flow in the cartilage space.

Phenotype of isolated MSCs and induced MSCs. MSCs can rapidly proliferate under suitable conditions with high proportion of FBS. When grown at low density, MSCs are capable of forming colonies. Also, they can differentiate into multiple lineages when induced by special induction medium. When isolated from bone marrow, primary MSCs showed colony-forming capacity (Fig. 3A) and the morphology of MSC colonies was also checked by crystal violet staining (Fig. 3B). After passaging, MSCs showed a fibroblast-like, spindle-shaped morphology and a homogeneous phenotype (data not shown). Then we used osteogenic and chondrogenic medium to induce MSCs. Induced osteogenic commitment cells showed typical flattened morphology after one week's induction (Fig. 3C). Also the osteogenic commitment differentiation was proved by alkaline phosphatase staining (Fig. 3D). Besides, chondrogenic MSCs were induced after 14 days' culture. The morphology was seen in the bright field picture (Fig. 3E) and also proved by Alcian Blue staining (Fig. 3F).

Effects of fluidic shear stress and osteoinduced MSCs on the viability and proliferation of cells. The bottom layer of the chip had three cell culture chambers, in which chondrocytes, chondroinduced MSCs and a mixture of them were seeded inside, respectively. Through the fluorescent probe of $\mathrm{DiI}$ (red) and $\mathrm{DiO}$ (green), the distribution of cells on the bottom layer could be clearly observed, which indicated the uniform distribution of cells in 3 separated chambers (Fig. 4A). Besides, the viability of cells in different groups was evaluated (Fig. 4B). Under different experiment conditions, all these cells exhibited good state after 


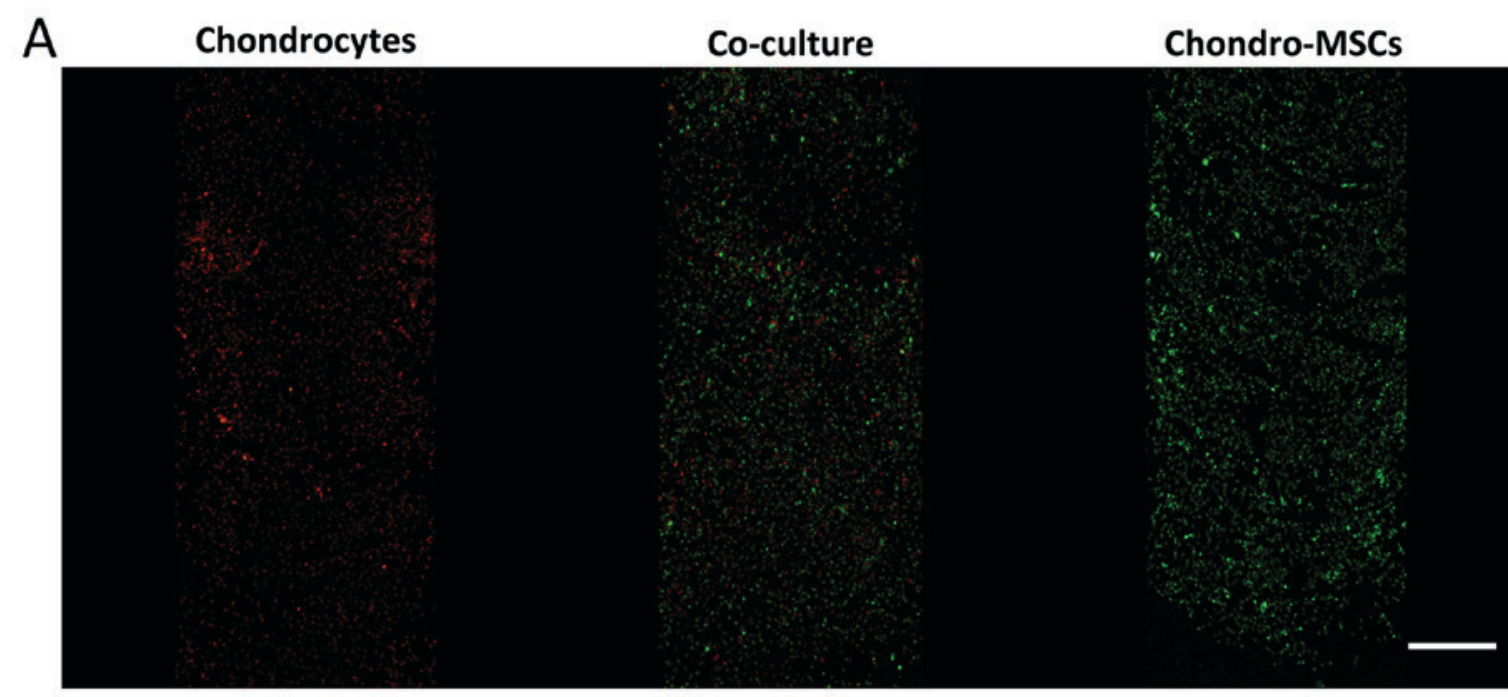

B

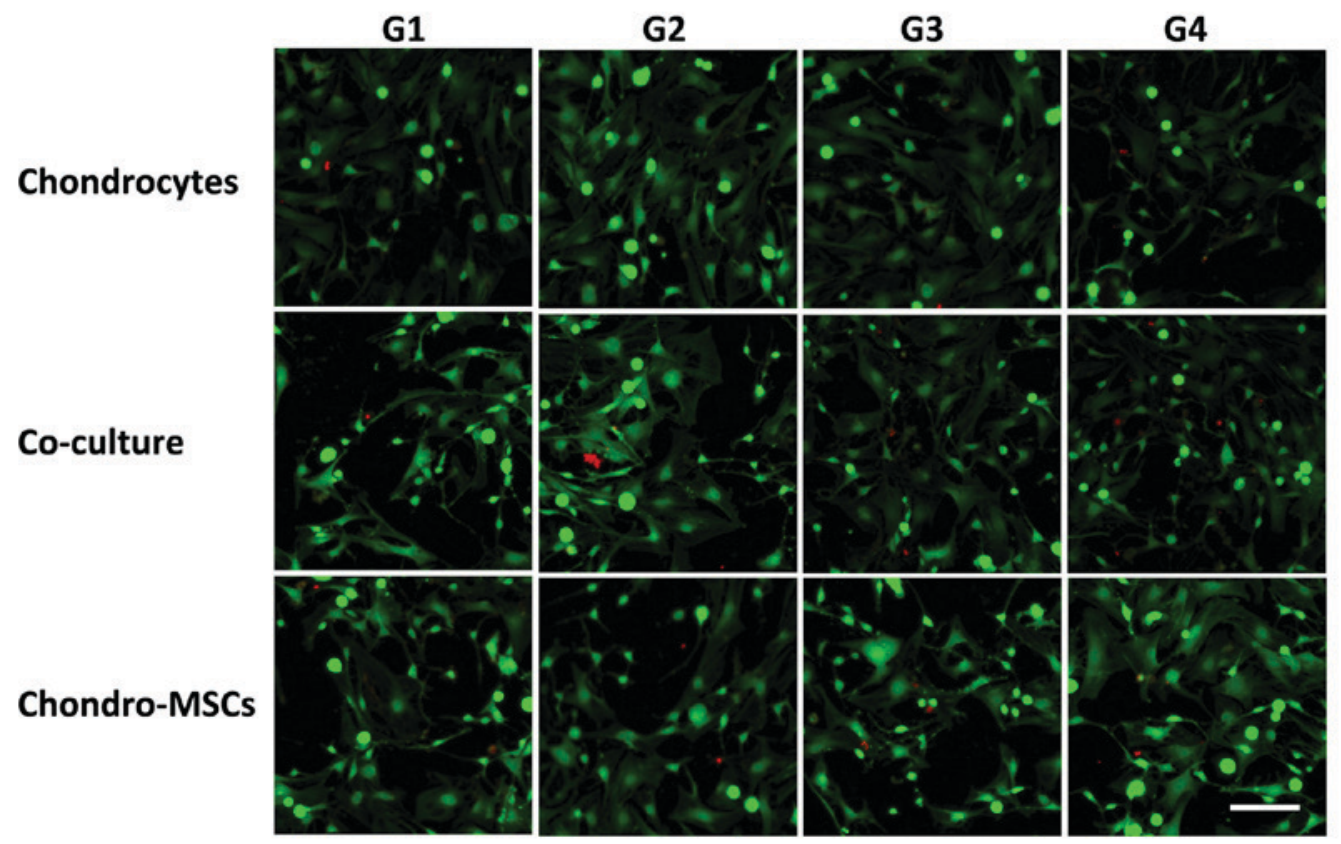

C
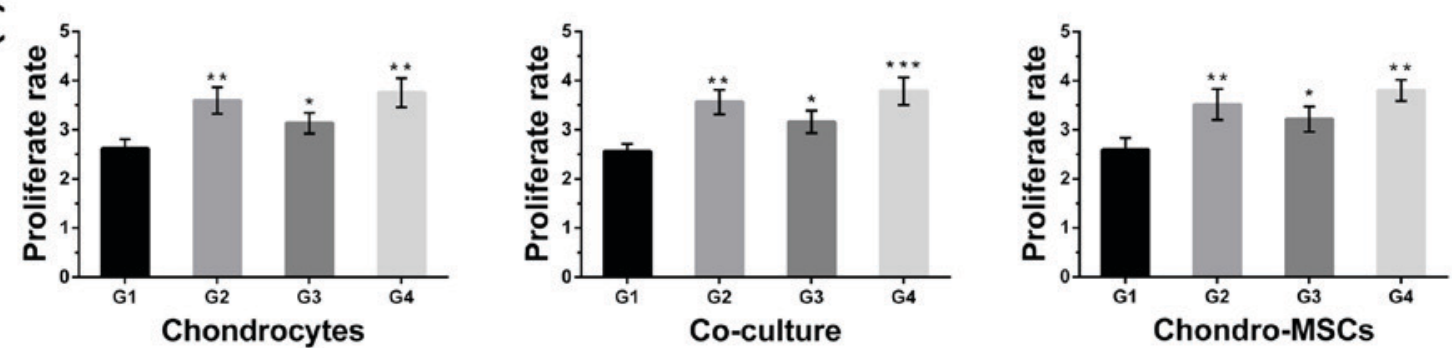

Figure 4. Evaluation of the viability and proliferation of chondrocytes and chondroinduced MSCs under different conditions. (A) Chondrocytes and chondroinduced MSCs stained with DiI (red) and DiO (green) were imaged. Scale bar is $500 \mu \mathrm{m}$. (B) Chondrocytes and chondroinduced MSCs transfected with green fluorescent protein (GFP, green) and PI staining (red) were imaged. Scale Bar is $100 \mu \mathrm{m}$. (C) The proliferation of chondrocytes and chondroinduced MSCs in four groups. The error bars are the mean \pm standard deviation. ${ }^{*} \mathrm{P}<0.05,{ }^{* *} \mathrm{P}<0.01,{ }^{* * *} \mathrm{P}<0.001$ vs. Group 1 . MSCs, mesenchymal stem cells.

7 days' culture. Very few dead cells (positive propidium iodide (PI) staining) were observed, which indicated the feasibility of this microfluidic chip for characterizing the stimulation of osteoinduced MSCs and the shear stress to the cells on the bottom layer. To analyze the effects of osteoinduced MSCs and fluid shear stress on cell proliferation, the proliferation rates of cells in different groups were analyzed by counting the cells (stained by DAPI, data not shown) attached to the bottom of the chambers. As shown in Fig. 4C, the proliferation rates of cells in group 2/3/4 were higher than the proliferation rate in group 1 . These findings suggested that both the given shear stress and the osteoinduced MSCs could affect the proliferation rates of chondroinduced MSCs, chondrocytes and a mixture of two cells.

Effects of shear stress and osteoinduced MSCs on the shape and morphology of cells. To characterize the shape and 
A

Chondrocytes

Co-culture

Chondro-MSCs
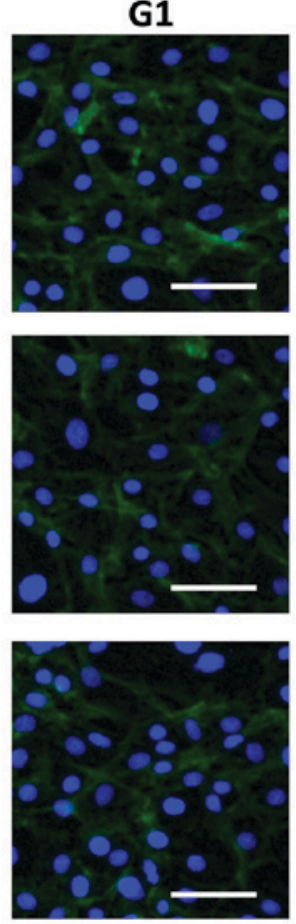

G2
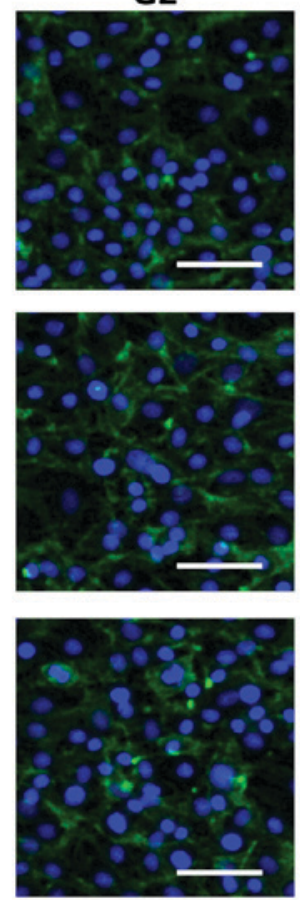

G3
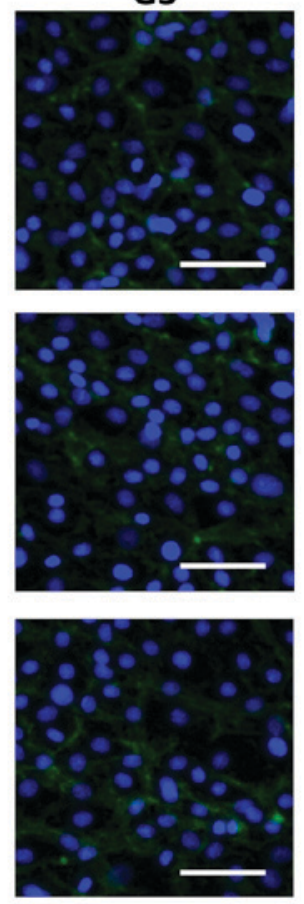
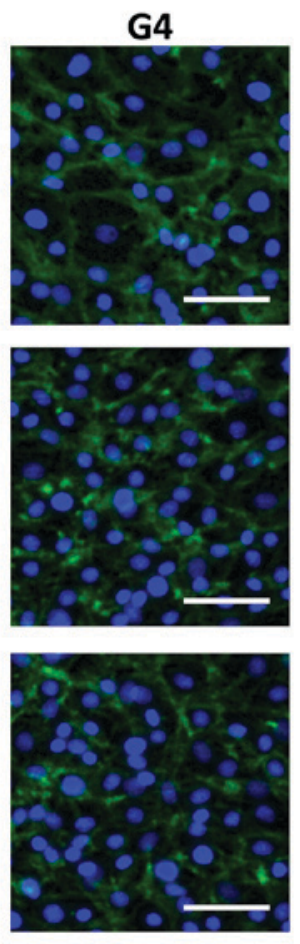
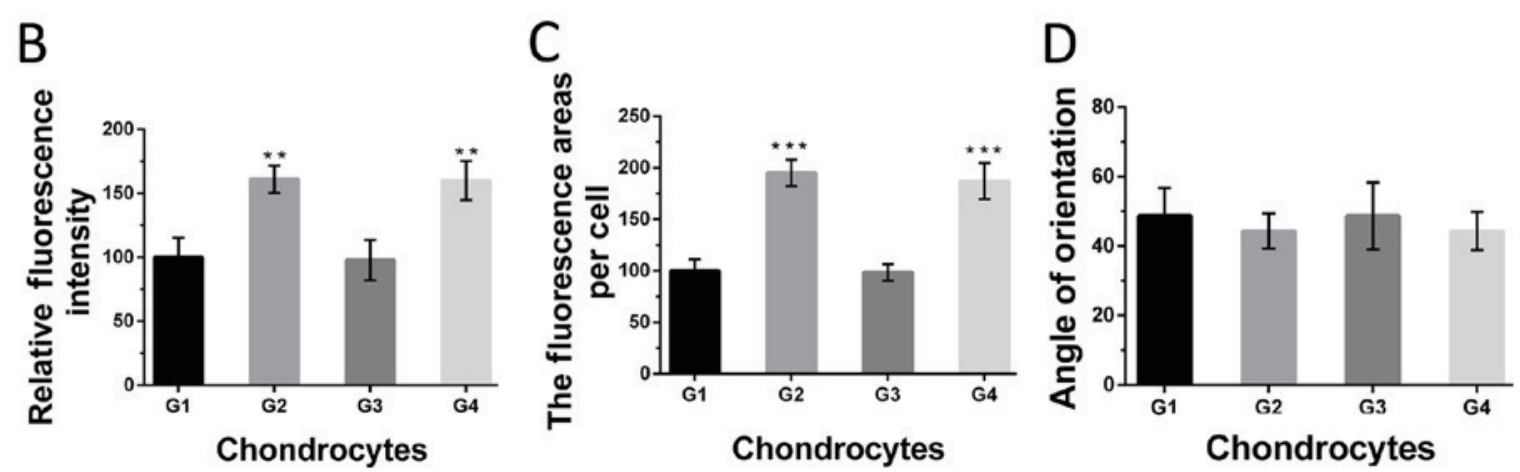

Figure 5. Morphological response of chondrocytes to distinct experiment conditions. (A) Chondrocytes and chondroinduced MSCs were immunostained for intermediate filament (green) and DAPI (blue). Scale bar is $100 \mu \mathrm{m}$. (B) Quantitative analysis of the relative fluorescence intensities under different conditions. (C) Histogram of the fluorescence areas per cell of intermediate filament expressed. (D) Cytoskeletal fiber orientation angles under different conditions. The error bars are the mean \pm standard deviation. ${ }^{* *} \mathrm{P}<0.01,{ }^{* * *} \mathrm{P}<0.001$ vs. Group 1 . MSCs, mesenchymal stem cells.

cytoskeleton change of cells on the bottom layer in response to the shear stress, the morphology change of chondroinduced MSCs, chondrocytes and a mixture of them was evaluated by intermediate filament staining (Fig. 5A). The results show that chondrocytes showed significant differences on intermediate filament expression under perfusion condition (group 2 and 4), compared with that in group 1 (Fig. 5B). Besides, chondrocytes culture in group 2, 4 showed remarkable morphological changes with spread adhering area per cell on the substrate of the chips (Fig. 5C). Similar results were observed for chondroinduced MSCs and the mixture of cells (data not shown). However, the angle of the orientation induced by the change of cytoskeleton showed no significant difference compared with those in group 1 (Fig. 5D), which indicated the fluid flow may be not strong enough to change the angle of cellular orientation.

Phenotypic variations of cells in response to shear stress and osteoinduced MSCs. After 7 days' cultivation, collagen I, collagen II and aggrecan, the markers for the dedifferentiated and differentiated phenotype of chondrocytes and chondroinduced MSCs, respectively, were characterized by immunofluorescence staining and GPCR to evaluate the phenotypic changes of cells in different groups. The detail data of the relative fluorescence intensities of collagen I, collagen II and aggrecan can be seen in Figs. 6-8. In terms of collagen I, the cells in group 1 were nearly negative (Fig. 6A). The mechanical loading provided by perfusion system in group 2 enhanced the expression of collagen I, while osteoinduced MSCs on the top layer weakened it. However, the cells in group 4, in which the effects of osteoinduced MSCs and the shear stress were combined, showed a weaker staining intensity compared with those in group 2, but stronger than those in group 3, indicating the rescue of the hyaline cartilage phenotype of cells by the stimulation of osteoinduced MSCs. This tendency can also be seen in qPCR result (Fig. 6B). Shear stress improved the mRNA expression of collagen I and this improvement can be reduced by stimulation of osteoinduced MSCs in Group 4 when compared with Group 2. 
A
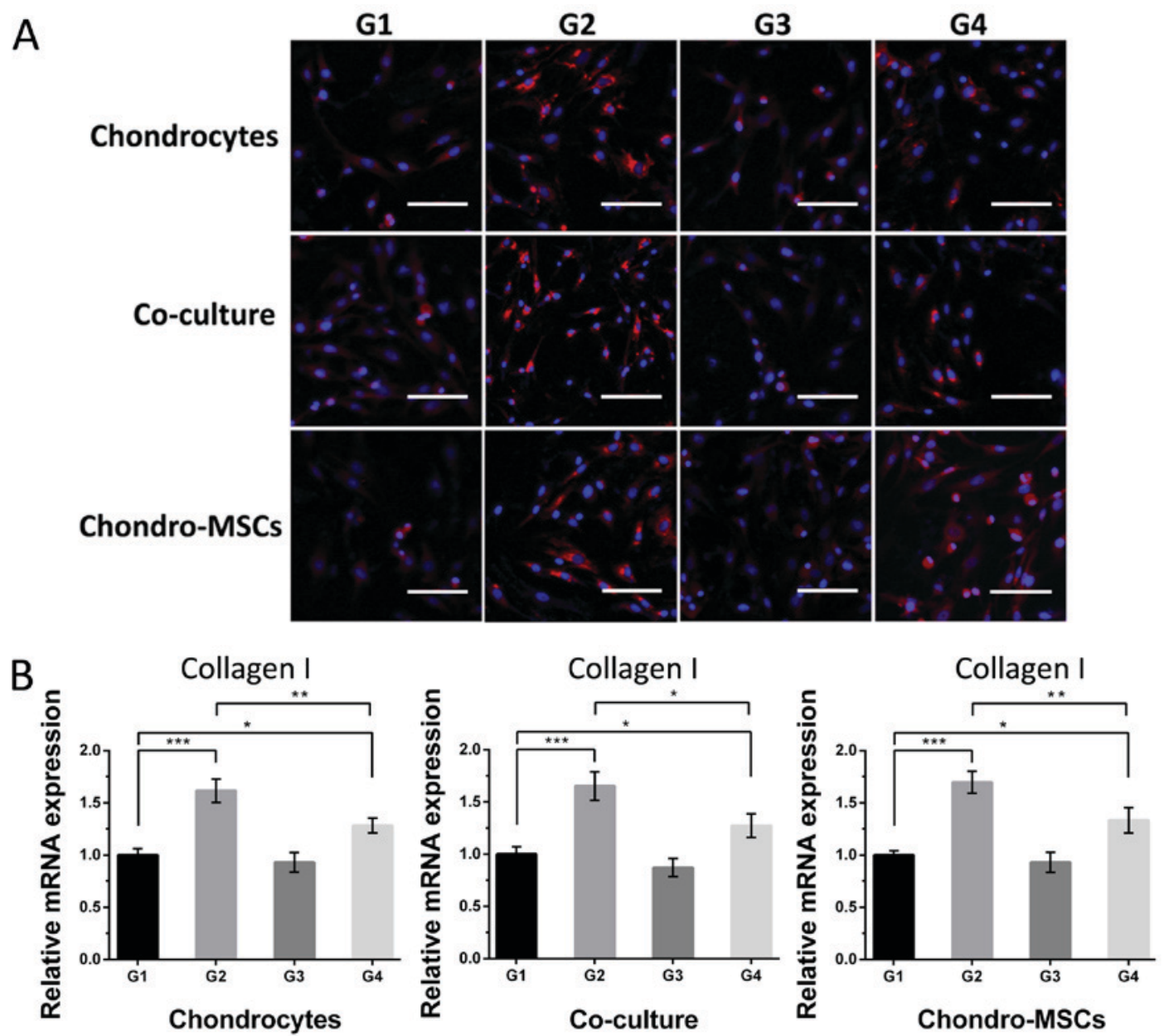

Figure 6. The phenotypic variations of chondrocytes and chondrogenically differentiate MSCs in response to different conditions. Cells in the buttom layer were immunostained for collagen I (red), and DAPI (blue) after 7 days of cultivation. (A) Fluorescence images of collagen I staining for cells under different conditions. Scale bar is $100 \mu \mathrm{m}$. (B) Comparisons of the relative mRNA expression of collagen I under different conditions using qPCR. The error bars are the mean \pm standard deviation. ${ }^{*} \mathrm{P}<0.05,{ }^{* * *} \mathrm{P}<0.01,{ }^{* * *} \mathrm{P}<0.001$ vs. Group 1 . MSCs, mesenchymal stem cells.

Then for collagen II and aggrecan, the cells in group 1 were weakly positive (Figs. 7A and 8A). As for cells in group 2 and 3, where there are perfusion systems on the bottom layer and osteoinduced MSCs on the top layer, the expression of collagen II and aggrecan was significantly higher than that in group 1. Cells in group 4, which had perfusion systems and osteoinduced MSCs simultaneously, the fluorescent intensity of collagen II and aggrecan was the strongest compared with those in other groups, indicating that osteoinduced MSCs on the top layer and the shear stress provided by the perfusion system acted synergistically to the cells on the bottom layer. Noncontact co-culture with osteoinduced MSCs neutralized the negative effect of the shear stress and even reversed the dedifferentiation process. Figs. 7B and 8B showed the changes of mRNA levels of collagen II and aggrecan in 4 groups. The collagen II and aggrecan mRNA expression were significantly up-regulated by shear stress and osteoinduced MSCs and the effects were synergistic.

\section{Discussion}

MSCs and chondrocytes are commonly used in cartilage and bone tissue engineering (9,21-23). MSCs can differentiate into osteogenic lineage in the bone scaffold and chondrogenic lineage in the cartilage scaffold. Hence, the great proliferative potential and the ability to adapt to different phenotypes in vivo make MSCs a good candidate for osteochondral tissue engineering. However, the results about the interactions between MSCs and chondrocytes were conflicted (24-26). It was reported that osteoinduced MSCs could promote the extracellular matrix production of chondrocytes, with the effect depending on the differentiation states of MSCs (15). Another study showed that chondroinduced MSCs within the chondral layer exhibited enhanced chondrogenic phenotype when combined with osteoinduced MSCs (16). However, the studies on the interactions between osteoinduced MSCs and other cells (chondrocytes and chondroinduced MSCs) were conducted under static condition. When cells (e.g., myocardial cell, chondrocytes) are subjected to the mechanical loading in vivo, the cytoskeleton of cells were remodeled by the shear stress, resulting in a better nutrient transportation $(27,28)$. In this study, we used a perfusion system to mimic the mechanical microenvironment in vivo to study the combined effect of the shear stress and non-contact co-culture of osteoinduced MSCs to chondrocytes and chondroinduced MSCs metabolism.

The mechanical stimuli are important to regulate cartilage metabolism and maintain chondrocytes function. 

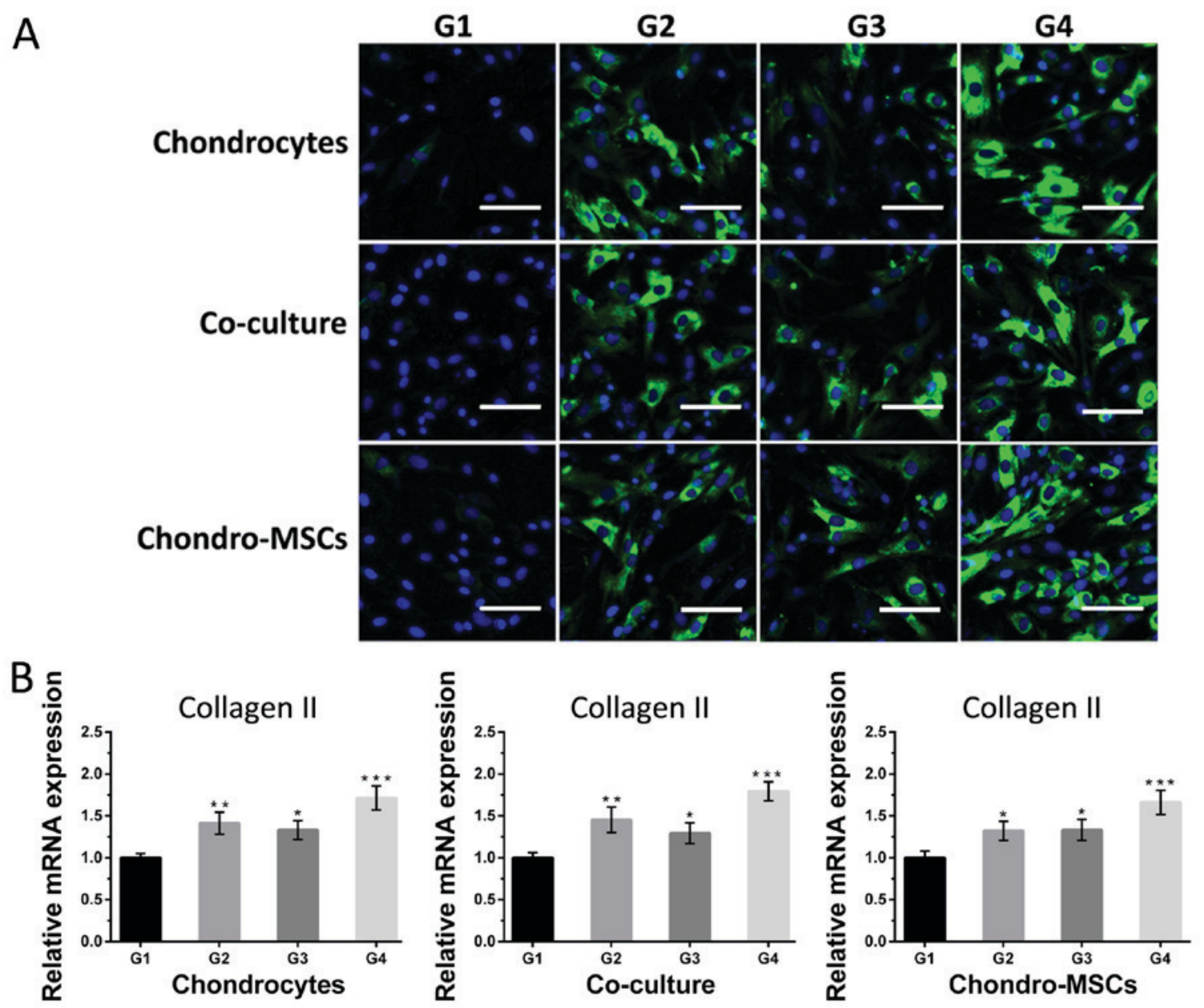

Figure 7. The phenotypic variations of chondrocytes and chondrogenically differentiate MSCs in response to different conditions. Cells in the buttom layer were immunostained for collagen II (green), and DAPI (blue) after 7 days of cultivation. (A) Fluorescence images of collagen II staining in cells after exposure to different conditions. Scale bar is $100 \mu \mathrm{m}$. (B) Comparisons of the relative mRNA expression of collagen II under different conditions using qPCR. The error bars are the mean \pm standard deviation. ${ }^{*} \mathrm{P}<0.05,{ }^{* * *} \mathrm{P}<0.01,{ }^{* * *} \mathrm{P}<0.001$ vs. Group 1 . MSCs, mesenchymal stem cells.

Microfabrication technology facilitates the regulation of the biological stimuli at the cellular and subcellular levels. In this work, we designed and fabricated a novel microchip by photo- and soft-lithographic approaches. Compared with conventional cell cultivation, this microfuidic chip not only reduced the cell sources, but also generated a controllable flow stimulus through tuning the input flow rate. Moreover, the transparent property of PDMS made the cells on the bottom layer observable and the semipermeable membrane between two layers allowed cytokines and small molecular compounds to freely transfer from the top layer to the bottom layer and prevented the cells from moving at the same time.

The three microchambers on the bottom layer were subjected to separated fluidic flow stress. The fluid shear stress on the cell surface can be calculated by the Poiseuille flow model in cylindrical microchamber. However, it is difficult to measure the fluid shear stress in the rectangular microchamber. Here we used hydromechanical CFD analysis to simulate the local fluid shear stress in three microchambers, and the results showed that the shear stress was uniform within the microchamber. Different perfusion systems were applied to achieve a range of shear stress, thus regulating the behaviors of MSCs and chondrocytes accordingly (29-31). The interstitial level of fluid in the intra-articular cartilage surface and different layers of articular surface was in the range of $10^{-5}$ to $10^{-2}$ dyne $/ \mathrm{cm}^{2}$ in the preceding researches $(32,33)$. In this work, the shear stress applied was $0.05 \mathrm{dyne} / \mathrm{cm}^{2}$, which was consistent with the level of interstitial fluid in the cartilage space.

Chondrocytes and chondroinduced MSCs are common cells used in tissue engineering. Chondroinduced MSCs are predifferentiated from MSCs and can facilitate the regeneration of chronic osteochondral lesions in vitro. One study showed that chondroinduced MSCs have superior regeneration ability compared with chondrocytes (34). Hence, in our study, we used both chondrocytes and chondroinduced MSCs to study the stimulation of osteoinduced MSCs under a fluid flow stimulus. The results showed that both chondrocytes and chondroinduced MSCs were similarly affected by osteoinduced MSCs, as well as their co-culture.

According to the results of our work, the proliferation rates of cells on the bottom layer were increased by the stimulus of shear stress on the bottom layer and the osteoinduced MSCs on the top layer. The proliferation rate of cells in group 4 showed the promotion effect was synergistic when the shear stress and osteoinduced MSCs were combined. Cytoskeleton, composed of intermediate filaments, actin and microtubules, is considered as a key mechanotransduction factor involved in 
A

Chondrocytes
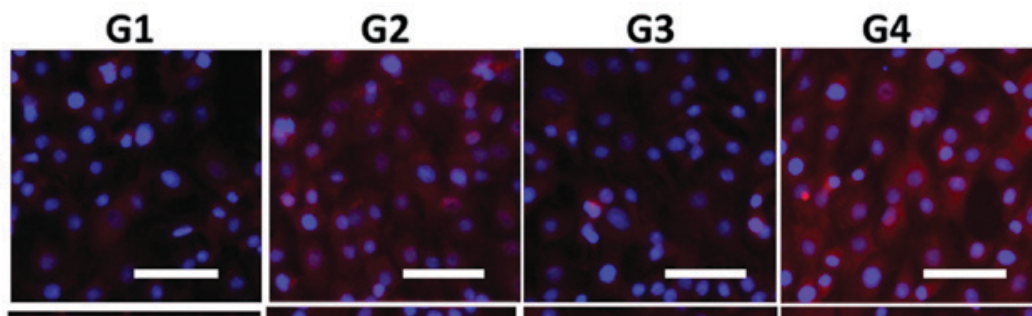

Co-culture
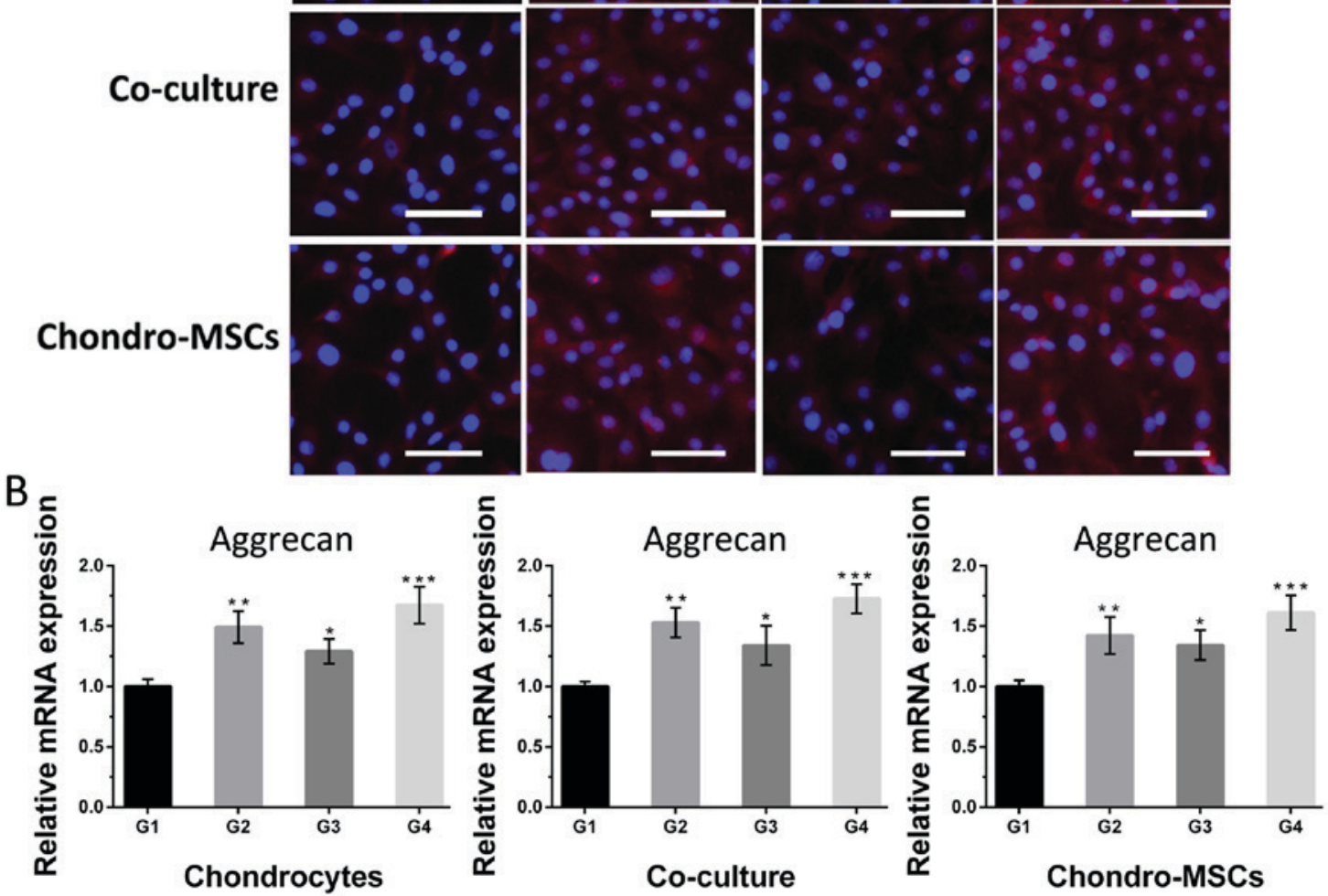

Figure 8. The phenotypic variations of chondrocytes and chondrogenically differentiate MSCs in response to different conditions. Cells in the buttom layer were immunostained for aggrecan (red), and DAPI (blue) after 7 days of cultivation. (A) Fluorescence images of aggrecan staining in cells after exposure to different conditions. Scale bar is $80 \mu \mathrm{m}$. (B) Comparisons of the relative mRNA expression of aggrecan under different conditions using qPCR. The error bars are the mean \pm standard deviation. ${ }^{*} \mathrm{P}<0.05,{ }^{* *} \mathrm{P}<0.01,{ }^{* * *} \mathrm{P}<0.001$ vs. Group 1 . MSCs, mesenchymal stem cells.

morphology change in response to flow stimulus. In this study, we evaluated the morphology change of cells by intermediate filament staining. The cells exhibited obvious morphological changes with flattened and increased adhering area per cell on the substrate. Besides, the mean area and IF staining expression of cells under perfusion condition were significant higher, indicating the remodeling of the cells' cytoskeleton under appropriate flow stimulus. However, the alignment of cells found in the previous work (35) was not observed in our work, suggesting that the level of the shear stress applied was not effective enough to cause the morphological change.

According to abovementioned results, chondrocytes showed phenotypic variation under the shear stress and the stimulation of osteoinduced MSCs. The increasing expression of collagen I in group 2 and 4 indicated the double-edged sword function of fluid shear stress. The results were in accordance with the previous studies $(36,37)$. Interestingly, the dedifferentiation effect brought by fluid shear stress could be neutralized by the stimulation of chondrocytes, highlighting the importance of osteoinduced MSCs in the maintaining of chondrocytes in tissue engineering. Excessive flow stimulus could elicit the proinflammatory cytokines release (38) and the matrix degradation of chondrocytes (39). However, the osteoinduced
MSCs may secrete one or several soluble factors to offset the inferior phenotypic variation induced by shear stress. In terms of collagen II and aggrecan, the expression in group 2 and 3 showed significantly increasing compared with group 1 . Besides, the expression of collagen II and aggrecan in group 4 was higher than that in group 2 and 3, demonstrating that not only both of the shear stress and osteoinduced MSCs could promote the differentiated phenotype of cells, but they also possessed synergistic effect on the differentiated phenotype of chondrocytes when the shear stress and the osteoinduced MSCs were combined.

Nevertheless, the cells on the bottom layer are not cultured in a $3 \mathrm{D}$ matrix in our work, which means only the upper surface of cells is exposed to the shear stress. Additionally, the soluble factors (e.g., BMPs, IGFs, FGF, and Endothelin-1) that mediate the 'reverse' process remain unknown. Further study will explore the exact cytokines that influence the phenotypic variation of cells. Furthermore, to better mimic the physiological properties in vivo, the microfluidic chip will be modified to an adoptable IIID cell culture on the bottom layer. Notwithstanding these limitations, this new designed lab-on-a-chip device can hurdle the obstacles of conventional method in the study of the stimulation of 
osteoinduced MSCs on chondrocytes metaobolism in a mechanical environment, which may help the development of clinical transplantation.

This study presented a novel integrated microfluidic chip to study the intercellular interactions between different cells in a fluid flow-induced mechanical environment. Based on the microfluidic chip, the morphological change, the proliferation rate and the phenotypic responses of chondroinduced MSCs, chondrocytes and the co-culture of them to osteoinduced MSCs in a mechanical microenvironment were investigated. The results showed both chondrocytes and chondroinduced MSCs were similarly affected by osteoinduced MSCs. The shear stress $\left(0.05 \mathrm{dyne} / \mathrm{cm}^{2}\right)$ and the osteoinduced MSCs were beneficial to maintain the phenotype of chondrocytes, and the effects were synergetic. Besides, the osteoinduced MSCs can reverse the dedifferentiate change induced by the fluid flow. The microfluidic chip allows us to better mimic the mechanical environment in vivo and minimizes the volume of cell resources down to a micron scale, providing a convenient evaluation tool in tissue engineering.

\section{Acknowledgements}

This study was supported by National Nature Science Foundation of China (nos. 91543121, 81573394 and 81273483), International Science and Technology Cooperation Program of China (no. 2015DFA00740), Special Fund for Agro-scientific Research in the Public Interest (no. 201303045), National scientific instrument development project (Chinese Academy of Sciences), Key Laboratory of Separation Science for Analytical Chemistry (Dalian Institute of Chemical Physics, Chinese Academy of Sciences).

\section{References}

1. Caron M, Emans P, Coolsen M, Voss L, Surtel D, Cremers A, van Rhijn LW and Welting TJ: Redifferentiation of dedifferentiated human articular chondrocytes: Comparison of 2D and 3D cultures. Osteoarthritis Cartilage 20: 1170-1178, 2012.

2. Hubka KM, Dahlin RL, Meretoja VV, Kasper FK and Mikos AG: Enhancing chondrogenic phenotype for cartilage tissue engineering: Monoculture and coculture of articular chondrocytes and mesenchymal stem cells. Tissue Eng Part B Rev 20: 641-654, 2014.

3. Zhang W, Zhuang A, Gu P, Zhou H and Fan X: A review of the three-dimensional cell culture technique: Approaches, advantages and applications. Curr Stem Cell Res Ther 11: 370-380, 2016.

4. Shin JW and Mooney DJ: Improving stem cell therapeutics with mechanobiology. Cell Stem Cell 18: 16-19, 2016.

5. Kon E, Robinson D, Verdonk P, Drobnic M, Patrascu JM, Dulic O, Gavrilovic G and Filardo G: A novel aragonite-based scaffold for osteochondral regeneration: Early experience on human implants and technical developments. Injury 47 (suppl 1): S27-S32, 2016.

6. Shimomura K, Moriguchi Y, Sugita N, Koizumi K and Yasui Y: Current strategies in osteochondral repair with biomaterial scaffold. In: Musculoskeletal research and basic science. Springer, Cham, pp387-403, 2016.

7. Pina S, Ribeiro V, Oliveira JM and Reis RL: Pre-clinical and clinical management of osteochondral lesions. In: Regenerative strategies for the treatment of knee joint disabilities. Springer, Cham, pp147-161, 2017.

8. Leach JK and Whitehead JR: Materials-directed differentiation of mesenchymal stem cells for tissue engineering and regeneration. ACS Biomater Sci Eng, 2017.

9. Vo T, Shah SR, Lu S, Tatara AM, Lee EJ, Roh TT, Tabata Y and Mikos AG: Injectable dual-gelling cell-laden composite hydrogels for bone tissue engineering. Biomaterials 83: 1-11, 2016.
10. Hung KC, Tseng CS, Dai LG and Hsu SH: Water-based polyurethane $3 \mathrm{D}$ printed scaffolds with controlled release function for customized cartilage tissue engineering. Biomaterials 83: 156-168, 2016.

11. Liu CH and Hwang SM: Cytokine interactions in mesenchymal stem cells from cord blood. Cytokine 32: 270-279, 2005.

12. Cawston TE: Metalloproteinase inhibitors and the prevention of connective tissue breakdown. Pharmacol Ther 70: 163-182, 1996.

13. Fujimoto E, Ochi M, Kato Y, Mochizuki Y, Sumen Y and Ikuta Y: Beneficial effect of basic fibroblast growth factor on the repair of full-thickness defects in rabbit articular cartilage. Arch Orthop Trauma Surg 119: 139-145, 1999.

14. Schinköthe T, Bloch W and Schmidt A: In vitro secreting profile of human mesenchymal stem cells. Stem Cells Dev 17: 199-206, 2008.

15. Lam J, Lu S, Meretoja VV, Tabata Y, Mikos AG and Kasper FK: Generation of osteochondral tissue constructs with chondrogenically and osteogenically predifferentiated mesenchymal stem cells encapsulated in bilayered hydrogels. Acta Biomater 10: 1112-1123, 2014

16. Rothenberg AR, Ouyang L and Elisseeff JH: Mesenchymal stem cell stimulation of tissue growth depends on differentiation state. Stem Cells Dev 20: 405-414, 2011.

17. Gao G, Zhang XF, Hubbell $K$ and Cui X: NR2F2 regulates chondrogenesis of human mesenchymal stem cells in bioprinted cartilage. Biotechnol Bioeng 114: 208-216, 2017.

18. Gosset M, Berenbaum F, Thirion S and Jacques C, Primary culture and phenotyping of murine chondrocytes. Nat Protoc 3: 1253-1260, 2008.

19. Green TR, Fisher J, Stone M, Wroblewski BM and Ingham E: Polyethylene particles of a 'critical size' are necessary for the induction of cytokines by macrophages in vitro. Biomaterials 19: 2297-2302, 1998

20. Livak KJ and Schmittgen TD: Analysis of relative gene expression data using real-time quantitative PCR and the 2(-Delta Delta C(T)) method. methods 25: 402-408, 2001.

21. Markstedt K, Mantas A, Tournier I, MartínezÁvila, Hc Hägg D and Gatenholm P: 3D bioprinting human chondrocytes with nanocellulose-alginate bioink for cartilage tissue engineering applications. Biomacromolecules 16: 1489-1496, 2015.

22. Schwarz S, Elsaesser AF, Koerber L, Goldberg-Bockhorn E, Seitz AM, Bermueller C, Dürselen L, Ignatius A, Breiter R and Rotter N: Processed xenogenic cartilage as innovative biomatrix for cartilage tissue engineering: effects on chondrocyte differentiation and function. J Tissue Eng Regen Med 9: E239-E251, 2015.

23. Mellor LF, Mohiti-Asli M, Williams J, Kannan A, Dent MR, Guilak F and Loboa EG: Extracellular calcium modulates chondrogenic and osteogenic differentiation of human adipose-derived stem cells: A novel approach for osteochondral tissue engineering using a single stem cell source. Tissue Eng Part A 21: 2323-2333, 2015.

24. Chen WH, Lai MT, Wu AT, Wu CC, Gelovani JG, Lin CT, Hung SC, Chiu WT and Deng WP: In vitro stage-specific chondrogenesis of mesenchymal stem cells committed to chondrocytes. Arthritis Rheum 60: 450-459, 2009.

25. Liu X, Sun H, Yan D, Zhang L, Lv X, Liu T, Zhang W, Liu W, Cao Y and Zhou G: In vivo ectopic chondrogenesis of BMSCs directed by mature chondrocytes. Biomaterials 31: 9406-9414, 2010.

26. Wu L, Prins HJ, Helder MN, van Blitterswijk CA and Karperien M: Trophic effects of mesenchymal stem cells in chondrocyte co-cultures are independent of culture conditions and cell sources. Tissue Eng Part A 18: 1542-1551, 2012.

27. Chen CY, Chiang TS, Chiou LL, Lee HS and Lin FH: 3D cell clusters combined with a bioreactor system to enhance the drug metabolism activities of C3A hepatoma cell lines. J Mater Chem B 4: 7000-7008, 2016.

28. Salinas M, Rath S, Villegas A, Unnikrishnan V and Ramaswamy S: Relative effects of fluid oscillations and nutrient transport in the in vitro growth of valvular tissues. Cardiovasc Eng Technol 7: 170-181, 2016.

29. Nie Z: Assessment of bioprocess shear stress as a tool to enhance osteogenic induction of mesenchymal cells (unpublished $\mathrm{PhD}$ thesis). University College London, 2017.

30. Sonam S, Sathe SR, Yim EK, Sheetz MP and Lim CT Cell contractility arising from topography and shear flow determines human mesenchymal stem cell fate. Sci Rep 6: 20415, 2016. 
31. Su YP, Chen CN, Chang HI, Huang KC, Cheng CC, Chiu FY, Lee KC, Lo CM and Chang SF: Low shear stress attenuates COX-2 expression induced by resistin in human osteoarthritic chondrocytes. J Cell Physiol 232: 1448-1457, 2017.

32. Park JY, Yoo SJ, Hwang CM and Lee SH: Simultaneous generation of chemical concentration and mechanical shear stress gradients using microfluidic osmotic flow comparable to interstitial flow. Lab Chip 9: 2194-2202, 2009.

33. Chen T, Buckley M, Cohen I, Bonassar L and Awad HA: Insights into interstitial flow, shear stress and mass transport effects on ECM heterogeneity in bioreactor-cultivated engineered cartilage hydrogels. Biomech Model Mechanobiol 11: 689-702, 2012.

34. Marquass B, Schulz R, Hepp P, Zscharnack M, Aigner T, Schmidt S, Stein F, Richter R, Osterhoff G, Aust G, et al: Matrix-associated implantation of predifferentiated mesenchymal stem cells versus articular chondrocytes: In vivo results of cartilage repair after 1 year. Am J Sports Med 39: 1401-1412, 2011.

35. Smith RL, Donlon BS, Gupta MK, Mohtai M, Das P, Carter DR, Cooke J, Gibbons G, Hutchinson N and Schurman DJ: Effects of fluid-induced shear on articular chondrocyte morphology and metabolism in vitro. J Orthop Res 13: 824-831, 1995.
36. Zhu F, Wang P, Lee NH, Goldring MB and Konstantopoulos K: Prolonged application of high fluid shear to chondrocytes recapitulates gene expression profiles associated with osteoarthritis. PLoS One 5: e15174, 2010.

37. Healy ZR, Lee NH, Gao X, Goldring MB, Talalay P, Kensler TW and Konstantopoulos K: Divergent responses of chondrocytes and endothelial cells to shear stress: Cross-talk among COX-2, the phase 2 response and apoptosis. Proc Natl Acad Sci USA 102: 14010-14015, 2005.

38. Luong L, Duckles H, Schenkel T, Mahmoud M, Tremoleda JL, Wylezinska-Arridge M, Ali M, Bowden NP, Villa-Uriol MC, van der Heiden $\mathrm{K}$, et al: Heart rate reduction with ivabradine promotes shear stress-dependent anti-inflammatory mechanisms in arteries. Throm Haemost 116: 181-190, 2016.

39. Trevino RL, Pacione CA, Malfait AM, Chubinskaya S and Wimmer MA: Development of a cartilage shear-damage model to investigate the impact of surface injury on chondrocytes and extracellular matrix wear. Cartilage 8: 444-455, 2017.

This work is licensed under a Creative Commons Attribution-NonCommercial-NoDerivatives 4.0 International (CC BY-NC-ND 4.0) License. 